\title{
SIMULADORES DE CIRCUITOS ELÉTRICOS: COMBINAÇÃO PARA UMA MAIOR EFICÁCIA NO ENSINO-APRENDIZAGEM
}

DOI: 10.37702/2175-957X.COBENGE.2021.3712

Frederico Silveira Madani - frederico.madani@gmail.com UNIP

Av. Engenheiro Francisco José Longo 1320

12245-001 - São José dos Campos - SP

Daniela Albuquerque Moreira Madani - daniela@unip.br UNIP

Rod. Pres. Dutra KM 157,5

12240-420 - Sao Jose dos Campos - SP

Fernando Silveira Madani - madani@ig.com.br

UNIP

Rod. Pres. Dutra, Km 157,5

12240-420 - Sao Jose dos Campos - SP

JOARES LIDOVINO DOS REIS JUNIOR - joares.junior@docente.unip.br UNIP

R: Maurício Cardoso 55

12236-495 - SÃO JOSÉ DOS CAMPOS - SP

Cristiano Roberto Martins Foli - crmfoli@yahoo.com.br

Universidade Paulista UNIP

Rua Manoel Bosco Ribeiro 817

12241-070 - São José dos Campos - SP

Eduardo Mikio Konigame - eduardokonigame@gmail.com

UNIP

Rod. Presidente Dutra km 157,5

12240-420 - Sao José dos Campos - SP

Resumo: As simulações computacionais são instrumentos importantes no processo de ensino-aprendizagem de circuitos elétricos. Elas podem constituir um elo entre os conceitos teóricos-matemáticos e a experiência prática. Nesse 
trabalho pesquisamos a percepção de alunos de Engenharia Elétrica com relação ao uso de dois simuladores livres para circuitos elétricos: Phet e TinkerCad. Nossos resultados mostram que para uma melhor eficácia no processo de aprendizagem é indicado o uso combinado e ordenado desses simuladores. Em uma primeira etapa, sugerimos usar o simulador Phet o qual proporciona uma abordagem mais conceitual e geral. Na sequência, sugerimos usar o simulador TinkerCad que possibilita um maior detalhamento dos conceitos de circuitos elétricos e, permite uma experiência de uso mais próxima com a realidade prática.

Palavras-chave: Simuladores; Circuitos Elétricos; Ensino em Engenharia. 


\section{SIMULADORES DE CIRCUITOS ELÉTRICOS: COMBINAÇÃO PARA UMA MAIOR EFICÁCIA NO ENSINO-APRENDIZAGEM}

\section{INTRODUÇÃO}

A importância de atividades experimentais no ensino de ciências e especificamente, na área de Engenharia é discutida a aproximadamente 300 anos (BORGES, 2002) (BARBERÁ \& VALDÉS, 1996)

No entanto, apesar de não ser a solução para todos os problemas no ensinoaprendizagem na área de engenharia (GALIAZZI, ROCHA, SCHMITZ, \& SOUZA, 2001) as atividades experimentais constituem um importante auxílio para a compreensão de conceitos.

No ano de 2018 foi proposto pela ABENGE o documento: Inovação na Educação em Engenharia - Proposta de Diretrizes Curriculares Nacionais para o Curso de Engenharia (ABENGE, 2018) o qual, entre várias propostas de competências a serem desenvolvidas em um curso de Engenharia, tem-se no segundo item: "Analisar e compreender os fenômenos físicos e químicos por meio de modelos matemáticos, computacionais ou físicos, validados por experimentação".

Nesse mesmo documento, também há a citação de propostas de estratégias e métodos a serem implantados pelas IES (Instituições de Ensino Superior), cita-se: "Processos de ensino / aprendizagem que contemplem métodos, técnicas e meios, sejam estes presenciais, remotos ou virtuais".

Assim, diante desses pontos colocados, entendemos a relevância de atividades experimentais no processo de ensino-aprendizagem em Engenharia.

No entanto, compreendemos que é necessário um vínculo entre conceitos teóricosmatemáticos e atividades práticas. Esse vínculo pode ser realizado através da simulação computacional (DORNELES \& ARAUJO, 2012) e (RONEN \& ELIAHU, 2000).

Nesse trabalho, pesquisamos o uso de dois simuladores computacionais: PhET Interactive Simulations (PHET, 2021) e TinkerCad (TinkerCad, 2021) para o estudo de circuitos elétricos.

O alvo da pesquisa foram 30 alunos do 3 ano de Engenharia, da modalidade Elétrica. Parte desses alunos já possuíam uma vivência prática profissional na área.

Assim, através da percepção desse grupo sobre o uso desses simuladores propomos uma abordagem que possa ser usada como um vínculo entre teoria e prática.

\section{METODOLOGIA}

Nesse trabalho nos guiamos pela proposta de Ausubel (AUSUBEL, 2003) que indica que os conceitos mais gerais da matéria de ensino, circuitos elétricos nesse estudo, deve ser primeiramente apresentado e, na sequência os detalhes e pormenores serem especificados.

Para Ausubel a organização de um conteúdo na mente de um aluno é uma estrutura hierárquica a qual as ideias mais gerais estão no nível superior e, de forma progressiva, conceitos mais diferenciados são acrescentados (DORNELES \& ARAUJO, 2012). 
Dentro dessa abordagem a metodologia adotada nesse estudo consistia em:

- Explicação teórica dos assuntos a serem abordados, com a escolha de circuitos elétricos resistivos em série e em paralelo (ALEXANDER \& SADIKU, 2013) (CAPUANO \& MARINO, 2002);

- Implementação das simulações através do Phet e do TinkerCad;

- Resposta a um questionário sobre a percepção, baseada na experiência de aprendizagem, do uso dos dois simuladores.

\section{Simuladores}

O Phet (PHET, 2021) é uma plataforma proposta pelo prêmio Nobel de Física Carl Wieman em conjunto com a Universidade do Colorado. Este portal disponibiliza um grupo de simulações que ajudam no ensino-aprendizagem de diversas ciências, inclusive Física e consequentemente Engenharia.

Essas simulações, muitas das vezes, são como laboratórios virtuais que permitem ao usuário relacionar conceitos teóricos com situações reais. O Phet é disponibilizado na Internet e pode ser usado de forma livre.

Essa plataforma de simulação pode ser acessada diretamente pela página da internet (ZARA, 2011) ou, caso o usuário queira, é possível instalar um aplicativo, inclusive para celulares.

Para esse trabalho usamos a simulação Circuit Construction Kit inserida na plataforma para a disciplina de Física.

O outro simulador usado em nossa pesquisa foi o TinkerCad (TinkerCad, 2021) que é uma ferramenta online de projetos de modelos 3D em Cad e, que também permite a simulação de circuitos elétricos: analógicos e digitais. O TinkerCad foi desenvolvido pela AutoDesk e, também pode ser usado de forma livre.

Para usar o TinkerCad no menu Circuitos é necessário que os alunos compreendam as formas de conexões elétricas do protoboard que é uma matriz de contato com furos de conexões condutoras. A grande vantagem de se utilizar o protoboard na prática é a facilidade de confecção do circuito uma vez que não é necessário a soldagem de componentes elétricos.

\section{Atividades propostas para os simuladores}

Após a explicação dos conceitos teóricos, foram propostos dois circuitos elétricos para serem simulados pelos alunos. Para cada um dos simuladores foram fornecidas sugestões de montagem.

Essas atividades tinham como objetivos:

- Verificar os conceitos relacionados a circuitos elétricos em série e em paralelo,

- Fornecer ao aluno uma visão do uso dos dois simuladores e,

- Quantificar a contribuição desses simuladores no processo de ensinoaprendizagem.

O primeiro conjunto de simulações consiste no estudo de um circuito elétrico em série. O diagrama elétrico desse circuito pode ser visto na Figura 1. 
Figura 1. Circuito Elétrico em série.

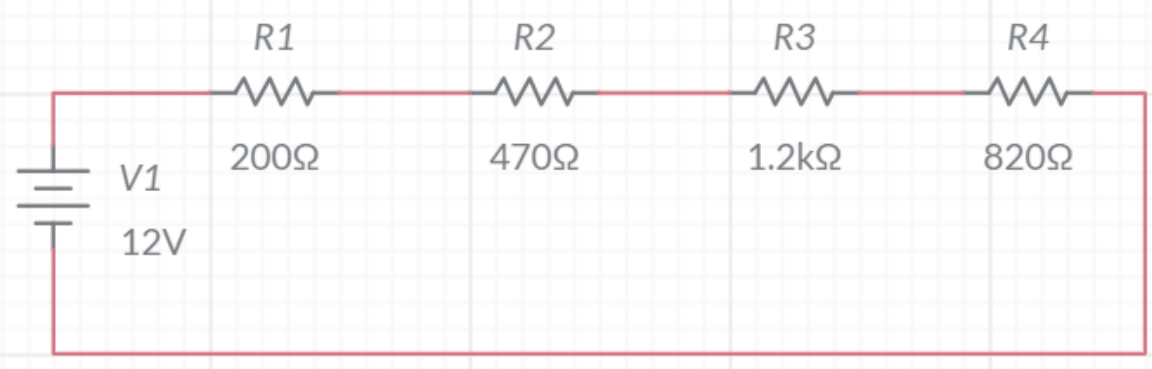

Fonte: Autores baseado em (CAPUANO \& MARINO, 2002). Figura 2.

A sugestão para a simulação desse circuito no Phet foi construída de acordo com a

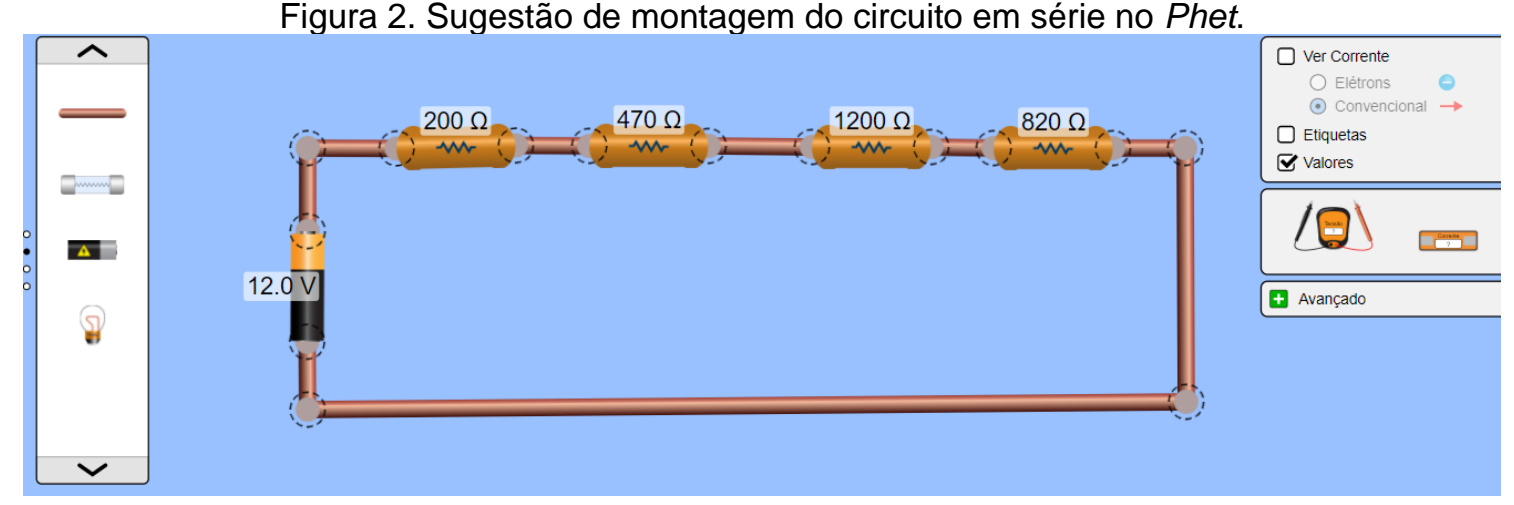

Fonte: Autores baseado em (CAPUANO \& MARINO, 2002)

A sugestão para a simulação desse circuito no TinkerCad foi construída de acordo com a Figura 3.

Figura 3. Sugestão de montagem do circuito em série no TinkerCad.

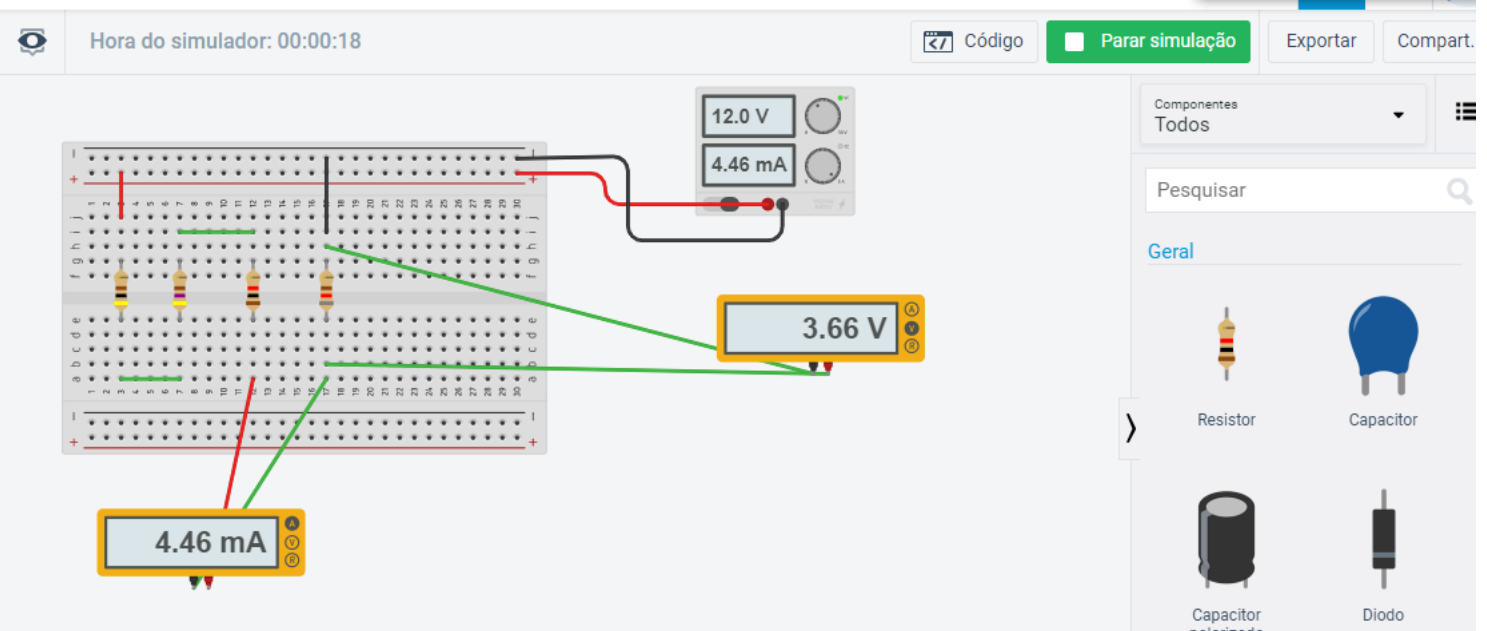

Fonte: Autores baseado em (CAPUANO \& MARINO, 2002).

Após a confecção dos circuitos nos dois simuladores, foi pedido aos alunos que medissem a intensidade de corrente elétrica, a tensão em cada elemento e preenchessem a Tabela 1 abaixo. Assim, poderiam verificar duas características elétricas fundamentais nesse tipo de circuito elétrico (ALEXANDER \& SADIKU, 2013) (CAPUANO \& MARINO, 2002):

- A intensidade de corrente elétrica é a igual para todos os elementos em série e, 
- A soma das tensões elétricas dos elementos em série é equivalente a tensão do gerador.

Tabela 1. Medidas elétricas do circuito em série realizada nos simuladores.

\begin{tabular}{l|l|l|l|l}
\hline$I_{R 1}(A)$ & $I_{R 2}(A)$ & $I_{R 3}(A)$ & $I_{R 4}(A)$ & $I_{T}(A)$ \\
\hline & \multicolumn{5}{|l}{} \\
\hline \multicolumn{5}{|l|}{} \\
\hline$R(\Omega)$ & 200 & 470 & 1200 & 820 \\
\hline$V(V)$ & & & & \\
\hline
\end{tabular}

Fonte: Autores baseado em (CAPUANO \& MARINO, 2002).

O segundo conjunto de simulações consiste no estudo de um circuito elétrico em paralelo. O diagrama elétrico desse circuito pode ser visto na Figura 4.

Figura 4. Circuito Elétrico em paralelo.

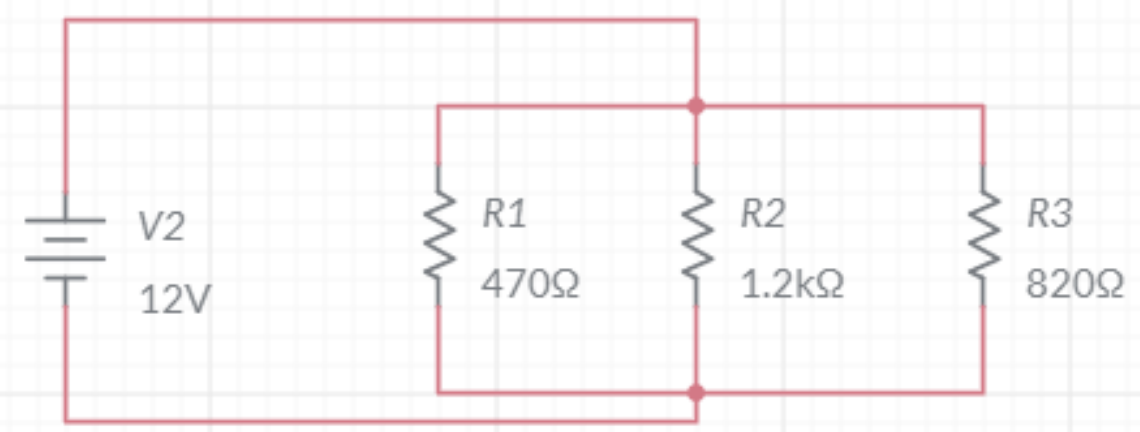

Fonte: Autores baseado em (CAPUANO \& MARINO, 2002). Figura 5.

A sugestão para a simulação desse circuito no Phet foi construída de acordo com a

Figura 5. Sugestão de montagem do circuito em paralelo no Phet.

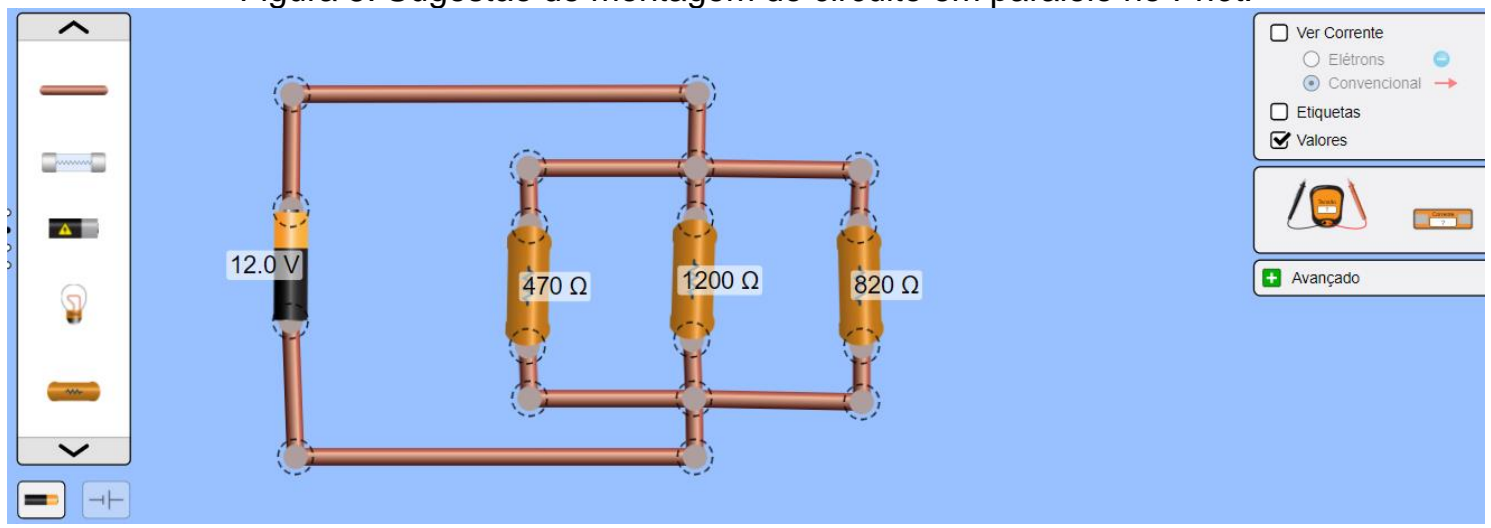

Fonte: Autores baseado em (CAPUANO \& MARINO, 2002).

A sugestão para a simulação desse circuito no TinkerCad foi construída de acordo com a Figura 6. 
Figura 6. Sugestão de montagem do circuito em série no TinkerCad.

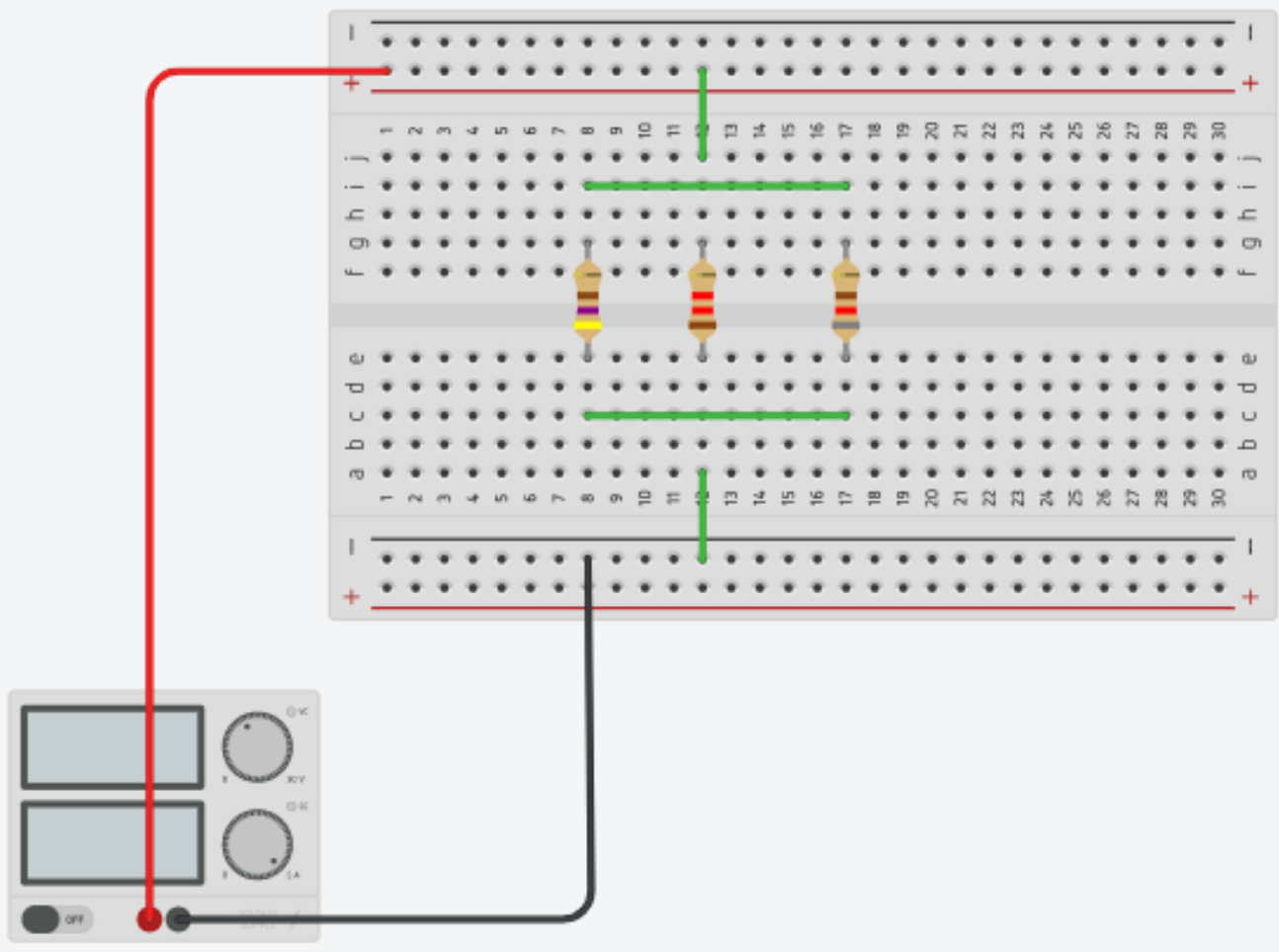

Fonte: Autores baseado em (CAPUANO \& MARINO, 2002).

Após a criação desse circuito resistivo em paralelo nos dois simuladores, foi requerido aos alunos que medissem a intensidade de corrente elétrica, a tensão elétrica em cada resistor e que preenchessem a Tabela 2. Logo, poderiam verificar as duas características elétricas fundamentais nesse tipo de circuito elétrico (ALEXANDER \& SADIKU, 2013) (CAPUANO \& MARINO, 2002):

- A soma da intensidade da corrente elétrica em cada elemento é igual a corrente fornecida pelo gerador $\mathrm{e}$,

- A tensão elétrica é a mesma em cada resistor.

Tabela 2. Medidas elétricas do circuito em paralelo realizada nos simuladores.

\begin{tabular}{l|l|l|l|l}
\hline$I_{R 1}(A)$ & $I_{R 2}(A)$ & $I_{R 3}(A)$ & $I_{R 4}(A)$ & $I_{T}(A)$ \\
\hline & & & & \\
\hline
\end{tabular}

\begin{tabular}{l|l|l|l|l}
\hline $\mathrm{R}(\Omega)$ & 200 & 470 & 1200 & 820 \\
\hline $\mathrm{V}(\mathrm{V})$ & & & & \\
\hline
\end{tabular}

Fonte: Autores baseado em (CAPUANO \& MARINO, 2002).

Após realizarem as simulações os alunos responderam a um questionário que buscava quantificar a percepção quanto ao uso desses simuladores. 


\section{RESULTADOS E DISCUSSÕES}

Apresentamos a seguir os resultados da percepção dos alunos com relação ao uso dos dois simuladores de circuitos elétricos.

O objetivo das duas primeiras perguntas era obter a percepção dos alunos com relação a dificuldade (facilidade) em usar os dois simuladores.

A primeira pergunta do questionário foi: "Você achou o simulador Phet - circuitos elétricos muito difícil de ser usado? Use a escala: considere 5, como muito difícil e 1 como muito fácil."

A segunda pergunta do questionário foi: "Você achou o simulador Tinkercad usado para circuitos elétricos muito difícil de ser usado? Use a escala; considere 5, como muito difícil e 1 como muito fácil".

O padrão de respostas para a primeira pergunta é apresentado no Gráfico 1 e, para a segunda pergunta no Gráfico 2.

Gráfico 1. Resultados para a primeira pergunta.

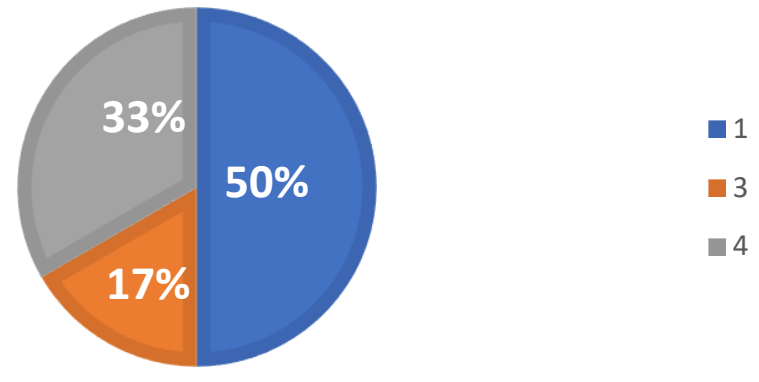

Fonte: Autores

Gráfico 2. Resultados para a segunda pergunta.

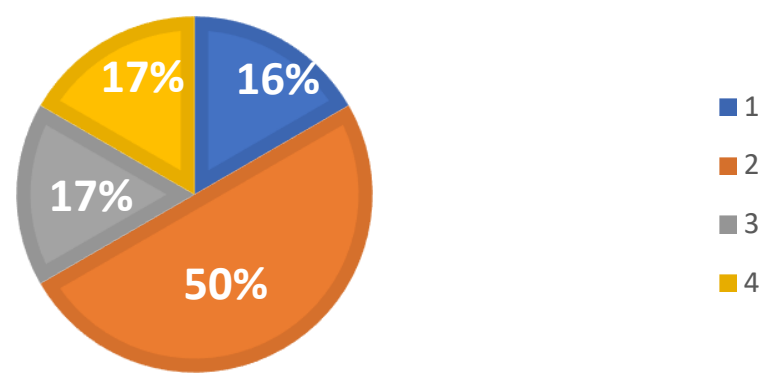

Fonte: Autores

De acordo com o percentual de respostas mostrado nas Figuras 7 e 8 e, considerando para a escala adotada que os valores 1 e 2 são "fáceis" (pouco difíceis), podemos afirmar que os dois simuladores são considerados de uso simples pelos alunos.

Essa característica é importante dentro do processo de ensino-aprendizagem por permitir que durante as atividades os alunos fiquem focados nos conceitos trabalhados.

O objetivo da terceira e quarta perguntas era obter a percepção dos alunos com relação ao auxílio em relação ao processo de aprendizagem dos conceitos de circuitos elétricos.

Esse conjunto de perguntas torna-se relevante uma vez que $60 \%$ alunos que responderam ao questionário possuem uma vivência profissional na área de circuitos elétricos. 
A terceira pergunta e quarta pergunta do questionário foram respectivamente:

- "Você considera, baseado em sua experiência prática de circuitos elétricos que o simulador Phet auxilia o aprendizado dos conceitos relacionados a circuitos elétricos? Use a escala: considere 5 como ajuda muito e 1 como ajuda pouco".

- "Você considera, baseado em sua experiência prática de circuitos elétricos, que o simulador Tinkercad auxilia o aprendizado dos conceitos relacionados a circuitos elétricos? Use a escala: considere 5 como ajuda muito e 1 como ajuda pouco".

O padrão de respostas para essas perguntas é apresentado nos Gráficos 3 e 4.

Gráfico 3. Resultados para a terceira pergunta.

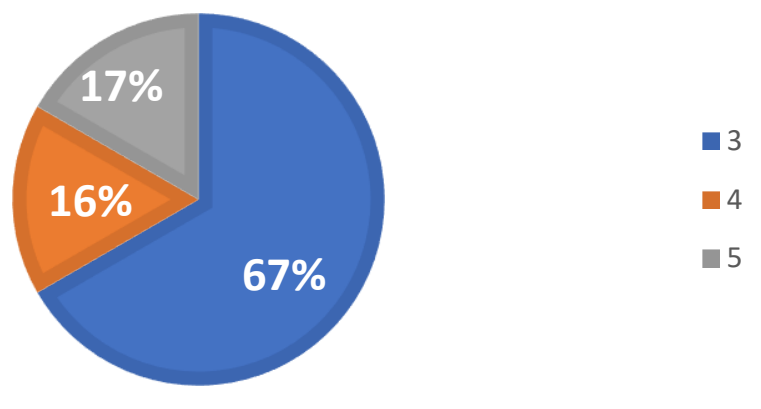

Fonte: Autores

Gráfico 4. Resultados para a quarta pergunta.

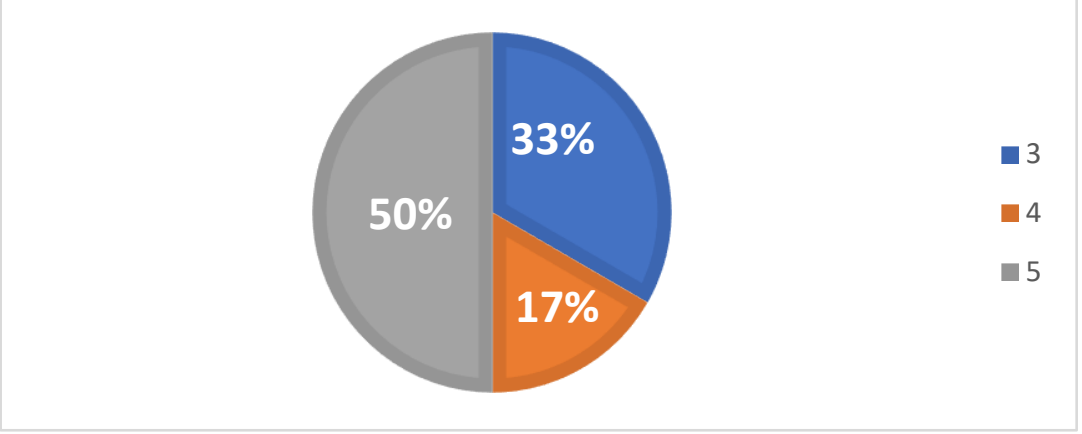

Fonte: Autores

Consideramos que os níveis 4 e 5 significam que os simuladores ajudam muito. Assim, analisando os resultados obtidos nos Gráficos 3 e 4, podemos afirmar que os alunos compreendem que os simuladores ajudam no processo de aprendizagem.

No entanto, existe uma diferença entre os TinkerCad em relação ao Phet. Para os alunos pesquisados o TinkerCad apresenta um percentual de $67 \%$ que consideram o seu uso proveitoso na aprendizagem. Mas, o percentual para o Phet é de $33 \%$.

Entendemos que essa diferença está relacionada ao fato de $60 \%$ do alunos já terem uma vivência profissional na área de circuitos elétricos e, o TinkerCad possuir uma interface mais próxima da prática real.

O próximo conjunto de perguntas teve como objetivo medir a percepção dos alunos para o quanto os dois simuladores aproximam da prática em termos reais.

Ressaltamos o fato que $60 \%$ dos alunos que responderam ao questionário já possuem uma prática real profissional em termos de circuitos elétricos. 
A quinta pergunta do questionário foi: "Você considera que o simulador Phet para circuitos elétricos, proporciona uma experiência de aprendizagem prática próxima ao real? Use a escala: considere 5 como muito próxima e 1 como distante".

A última (sexta) pergunta do questionário foi: "Você considera que o simulador Tinkercad para circuitos elétricos, proporciona uma experiência de aprendizagem prática próxima ao real? Considere 5 como muito próxima e 1 como distante".

O percentual de respostas a essas perguntas é apresentado nos Gráfico 5 e 6.

Gráfico 5. Resultados para a quinta pergunta.

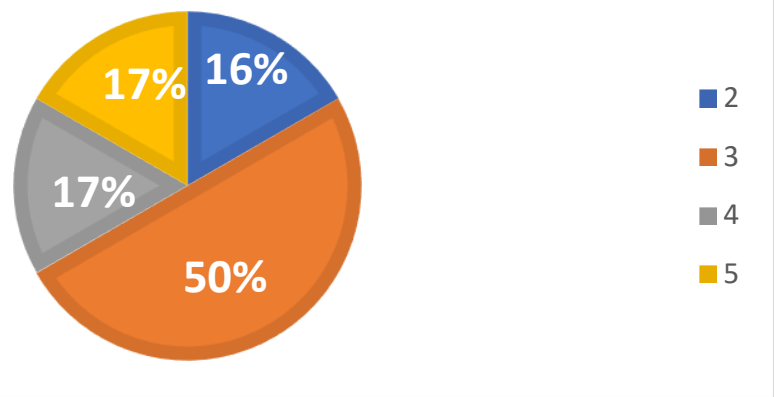

Fonte: Autores

Gráfico 6. Resultados para a sexta pergunta.

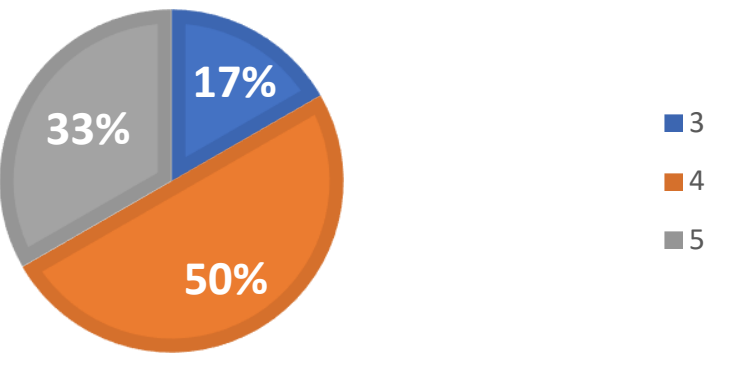

Fonte: Autores

Considerando os níveis 4 e 5 como próximos ao real, os Gráficos 5 (para o Phet) e 6 (para o TinkerCad) apresentam resultados distintos.

De acordo com a Figura 11, para $34 \%$ dos alunos o simulador Phet apresenta uma experiência próxima do real.

Esse resultado está provavelmente relacionado a interface gráfica do simulador. Apesar dessa interface ser de fácil uso (Gráfico 1) e auxiliar na aprendizagem de conceitos de circuitos elétricos (Gráfico 3), ela não permite uma experiência próxima do real no sentido de "não simular" em termos práticos a confecção de um circuito elétrico.

No entanto, de acordo com a Gráfico 6, para 83\% dos alunos, o simulador TinkerCad possibilita uma experiência próxima ao real.

Como $60 \%$ dos alunos tinham uma experiência prática de circuitos elétricos, que está relacionada a montagem em protoboard, para esses alunos o TinkerCad possibilita uma interface gráfica que "simula" em termos práticos a confecção de um circuito elétrico.

\section{Conclusões}

As atividades práticas são fundamentais no processo de ensino-aprendizagem na área de Engenharia. 
No entanto, as simulações podem também ser um recurso valioso nesse processo.

Nossos resultados indicam que os dois simuladores pesquisados (Phet e TinkerCad) contribuem para o processo de aprendizagem na área de circuitos elétricos.

Nossa pesquisa indicou que o TinkerCad possibilita uma aprendizagem mais próxima do real quando comparada com o Phet.

Assim, baseado em nossos resultados, concluímos que para uma maior eficácia do uso de simuladores para circuitos elétricos seria interessante estabelecer uma ordem de uso.

Para uma primeira abordagem dos conceitos de circuitos elétricos, o simulador Phet seria o adequado. Esse simulador, de uso simples, pode auxiliar a compreensão de conceitos mais gerais.

No entanto, para uma abordagem mais detalhada, o TinkerCad torna-se o mais adequado. Isso se deve ao fato que esse simulador permite uma experiência mais próxima da prática real para o aluno.

Assim, ao invés de escolher usar apenas um dos simuladores, indicamos o uso combinado e organizado para proporcionar uma maior eficácia no processo de ensinoaprendizagem.

Esse uso combinado dos dois simuladores podem ser uma vínculo entre os conceitos teóricos-matemáticos de circuitos elétricos e a experiência prática real.

\section{Agradecimentos}

Os autores agradecem aos alunos do 3 ano de Engenharia, na modalidade Elétrica, por terem participado nessa pesquisa.

\section{REFERÊNCIAS}

ABENGE. (2018). Inovação na Educação em Engenharia - Proposta de Diretrizes Curriculares Nacionais para o Curso de Engenharia . Brasília.

Alexander, C. K., \& Sadiku, M. N. (2013). Fundamentos de Circuitos Elétricos (5 ed.). New York: The McGraw-Hill Companies.

AUSUBEL, D. P. (2003). Aquisição e retenção de conhecimentos: uma perspectiva cognitiva. Lisboa: Plátano.

BORGES, A. T. (2002). Cad. Bras. Ens. Fís., p. 291.

CAPUANO, F., \& MARINO, M. (2002). Laboratório de eletricidade e Eletrônica. São Paulo: Érica.

Dorneles, P. F., \& Araujo, I. S. (2012). Ciência \& Educação, 18, p. 99.

GALIAZZI, M., ROCHA, J., SCHMITZ, L., \& SOUZA, M. (2001). Ciência \& Educação, 7, p. 249.

O. Barberá e P. Valdés. (1996). Enseñanza de las Ciencias, 14, p. 365. 
Phet. (2021). https://phet.colorado.edu/pt_BR/. Fonte: PhET - Physics Educational Technology.

RONEN, M., \& ELIAHU, E. (2000). Journal of Computer Assisted Learning, 16, p. 14.

TinkerCad. (2021). https://www.tinkercad.com/teach. Fonte: AutoDesk TinkerCad - .

Zara, R. A. (2011). Reflexão sobre a eficácia do uso de um ambiente virtual no. I/ ENINED - Encontro Nacional de Informática e Educação. Cascável, Pr.

\title{
SIMULATORS OF ELECTRICAL CIRCUITS: COMBINATION FOR MORE EFFECTIVENESS IN TEACHING-LEARNING
}

\begin{abstract}
The computational simulations are important tools in the process of teachinglearning electric circuits. They can be a link of theoretical concepts and practice experience. In this work we researched the perception of Electric Engineer students related of two free electric simulators: Phet and TinkerCad. Our results show that to improve the effectiveness of learning is indicated the tidy and combined use of these simulators. At first stage, we suggest using Phet that provides a conceptual approach. After, we suggest using TinkerCad that leads to a more detailed aspects of electric circuits and allow to a experience close to practical reality.
\end{abstract}

Keywords: Simulators. Electric circuits. Engineering teaching 